Modern Physics Letters A

(C) World Scientific Publishing Company

\title{
ASYMMETRIC EMBEDDING IN BRANE COSMOLOGY
}

\author{
YURI SHTANOV* and ALEXANDER VIZNYUK ${ }^{\dagger}$ \\ Bogolyubov Institute for Theoretical Physics, Kiev 03680, Ukraine \\ *shtanov@bitp.kiev.ua \\ †viznyuk@bitp.kiev.ua \\ LUIS NORBERTO GRANDA \\ Departamento de Física, Universidad del Valle, A.A. 25360, Cali, Colombia \\ ngranda@univalle.edu.co
}

\begin{abstract}
We derive a system of cosmological equations for a braneworld with induced curvature which is a junction between several bulk spaces. The permutation symmetry of the bulk spaces is not imposed, and the values of the fundamental constants, and even the signatures of the extra dimension, may be different on different sides of the brane. We then consider the usual partial case of two asymmetric bulk spaces and derive an exact closed system of scalar equations on the brane. We apply this result to the cosmological evolution on such a brane and describe its various partial cases.
\end{abstract}

Keywords: braneworld model

PACS Nos.: 04.50.+h, 98.80.Es

\section{Introduction}

The idea that our four-dimensional world can be described as a timelike hypersurface (brane) embedded in or bounding a five-dimensional manifold continues to be in the focus of modern investigations. Especially this concerns cosmological braneworld solutions which exhibit many interesting and unusual properties (see Refs. 1 3 for recent reviews). Theories with the simplest generic action involving scalar-curvature terms both in the bulk and on the brane allow for the possibilities of superacceleration of the universe expansion $\frac{415}{75}$ cosmological loitering even in a spatially flat universe, $\frac{6}{6}$ and "cosmic mimicry," erty that the braneworld model at low redshifts is virtually indistinguishable from the LCDM $(\Lambda+$ Cold Dark Matter) cosmology but has renormalized value of the cosmological density parameters. The possibility of explaining other dark-matter phenomena in such models is discussed in Ref. 8 ,

A vast majority of papers on braneworld cosmology are dealing with the situation of the $Z_{2}$ symmetry of reflection with respect to the brane, which is equivalent to the brane being the boundary of the bulk. The present letter is devoted to the investigation of solutions where such symmetry is not imposed. 
Braneworld cosmologies without $Z_{2}$ symmetry of reflection were a subject of investigation, for example, in Refs. 9 19, In these papers, however, the curvature term in the action on the brane was absent. The braneworld model without $Z_{2}$ symmetry was studied in Ref. 20 in the presence of the curvature term on the brane and with equal bulk cosmological and gravitational constants on either side of the brane. In Ref. 21, the $Z_{2}$-asymmetric model was considered in the braneworld theory with curvature term on the brane and with different cosmological constants in the bulk but with equal bulk gravitational constants on either sides of the brane. In a recent paper, 22 an asymmetric braneworld cosmology was under consideration in the model without the induced-curvature term on the brane but with different bulk cosmological and gravitational constants on either side of the brane.

In this letter, we would like to consider the most generic braneworld model in which the values of the bulk fundamental (gravitational and cosmological) constants are different on the two sides of the brane and, in addition to this, the signatures of the extra dimension on the two sides of the brane may be arbitrary (and different). Note that the $Z_{2}$-symmetric braneworld cosmology with timelike extra dimension was previously considered in Refs. 16, 23, 25,

Before considering the asymmetric case with a brane separating between two bulk spaces, we consider a generalization in which a brane is a multivolume junction, i.e., a boundary of $N$ bulk spaces with natural junction conditions. The theory of such spaces was initiated in Ref. 26. In the next section, we derive a system of cosmological equations for this configuration without assuming any symmetry between the bulk spaces. In particular, the values of the fundamental constants, and even the signatures of the extra dimension, may be different on different sides of the brane. After that, we study in more detail the usual case of two bulk spaces.

\section{A brane as a multivolume junction}

Our starting setup is a four-dimensional hypersurface (brane) $\mathcal{B}$ which is simultaneously a boundary of $N$ five-dimensional Riemannian manifolds (bulk spaces) $\mathcal{M}_{1}, \ldots, \mathcal{M}_{N}$ and has nondegenerate Lorentzian induced metric (see Fig. 1). The primary junction condition which makes the brane the boundary of all bulk spaces is that the induced metric is one and the same in all $\mathcal{M}_{I}, I=1, \ldots, N$. We assume that the bulk gravitational and cosmological constants on different sides of the brane can be different. The action of the theory has the form

$$
S=\sum_{I=1}^{N} M_{I}^{3}\left[\int_{\mathcal{M}_{I}}\left(\mathcal{R}_{I}-2 \Lambda_{I}\right)-2 \epsilon_{I} \int_{\mathcal{B}} K_{I}\right]+\int_{\mathcal{B}}\left(m^{2} R-2 \sigma\right)+\int_{\mathcal{B}} L\left(h_{a b}, \phi\right) .
$$

Here, $\mathcal{R}_{I}$ is the scalar curvature of the five-dimensional metric $g_{a b}^{I}$ on $\mathcal{M}_{I}$, and $R$ is the scalar curvature of the induced metric $h_{a b}=g_{a b}-n_{a}^{I} n_{b}^{I}$ on $\mathcal{B}$, where $n_{I}^{a}$ is the vector of the inner unit normal to the brane The quantity $K_{I}=K_{a b}^{I} h^{a b}$ is the

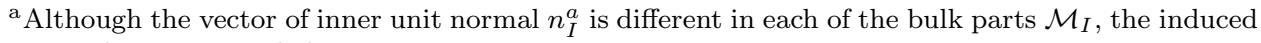
metric $h_{a b}$ is one and the same. 


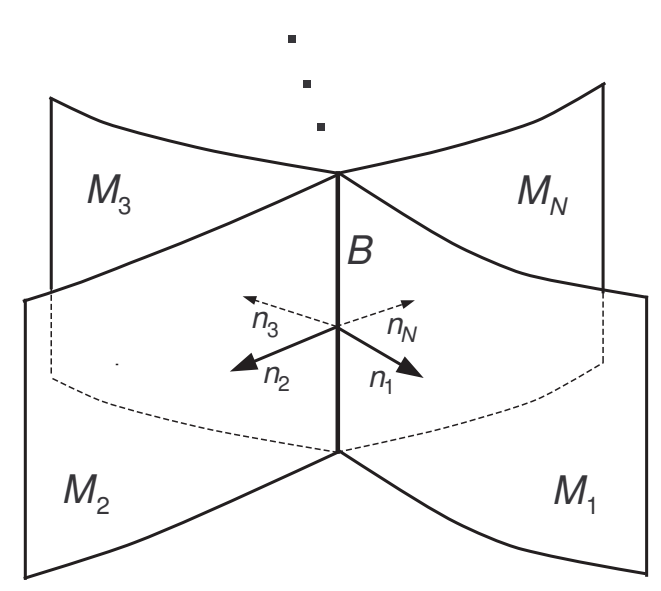

Fig. 1. Brane $\mathcal{B}$ as a junction between the bulk spaces $\mathcal{M}_{1}, \ldots, \mathcal{M}_{N}$.

trace of the symmetric tensor of extrinsic curvature $K_{a b}^{I}$ of $\mathcal{B}$ in $\mathcal{M}_{I}$. The parameter $\epsilon_{I}=1$ if the signature of the corresponding bulk part $\mathcal{M}_{I}$ is Lorentzian, so that the extra dimension is spacelike, and $\epsilon_{I}=-1$ if its signature is $(-,-,+,+,+)$, so that the extra dimension is timelike. The symbol $L\left(h_{a b}, \phi\right)$ denotes the Lagrangian density of the four-dimensional matter fields $\phi$ the dynamics of which is restricted to the brane $\mathcal{B}$ so that they interact only with the induced metric $h_{a b}$. All integrations over $\mathcal{M}_{I}$ and over $\mathcal{B}$ are taken with the corresponding natural volume elements. The symbols $M_{I}, I=1, \ldots, N$, denote the Planck masses of the corresponding spaces, $\Lambda_{I}, I=1, \ldots, N$, are the corresponding five-dimensional cosmological constants, and $m$ and $\sigma$ are the Planck mass and tension of the brane, respectively.

In this letter, we systematically use the notation and conventions of Ref. 27, In particular, we use the one-to-one correspondence between tensors in $\mathcal{B}$ and tensors in $\mathcal{M}$ which are invariant under projection to the tangent space to $\mathcal{B}$, i.e., tensors $T^{a_{1} \cdots a_{k}} b_{1} \cdots b_{l}$ such that

$$
T_{b_{1} \cdots b_{l}}^{a_{1} \cdots a_{k}}=h_{c_{1}}^{a_{1}} \cdots h^{a_{k}}{ }_{c_{k}} h_{b_{1}}{ }^{d_{1}} \cdots h_{b_{l}}{ }^{d_{l}} T^{c_{1} \cdots c_{k}}{ }_{d_{1} \cdots d_{l}} .
$$

Variation of action (11) gives the equation of motion in the five-dimensional bulk parts $\mathcal{M}_{I}$ :

$$
\mathcal{G}_{a b}^{I}+\Lambda_{I} g_{a b}^{I}=0, \quad I=1, \ldots, N,
$$

and on the brane $\mathcal{B}$ :

$$
m^{2} G_{a b}+\sigma h_{a b}=\sum_{I=1}^{N} \epsilon_{I} M_{I}^{3} S_{a b}^{I}+\tau_{a b},
$$

where $\mathcal{G}_{a b}^{I}$ and $G_{a b}$ are the Einstein's tensors of the corresponding spaces, $S_{a b}^{I} \equiv$ $K_{a b}^{I}-K^{I} h_{a b}$ is formed from the tensor of extrinsic curvature, and $\tau_{a b}$ denotes the 
four-dimensional stress-energy tensor of matter on the brane. It is the presence of the tensors $S_{a b}^{I}$ in the equation of motion (4) that makes the inner dynamics on the brane $\mathcal{B}$ unusual $b$

The Codazzi relations on the brane, in view of the bulk equation (3), read

$$
D^{a} S_{a b}^{I}=\mathcal{R}_{c d}^{I} n_{I}^{d} h^{c}{ }_{b}=0, \quad I=1, \ldots, N,
$$

where $D^{a}$ is the (unique) derivative on the brane $\mathcal{B}$ associated with the induced metric $h_{a b}$. Equation (4) then implies the relation

$$
D_{a} \tau_{b}^{a}=0 \text {. }
$$

Thus, the four-dimensional stress-energy tensor is covariantly conserved on the brane, which is a consequence of the absence of matter in the bulk.

Generalizing the procedure of Refs. 29,30 to the case of $N$ bulk spaces, we consider the Gauss identity:

$$
R_{a b c d}=h_{a}{ }^{f} h_{b}{ }^{g} h_{c}{ }^{k} h_{d}{ }^{j} \mathcal{R}_{f g k j}^{I}+\epsilon_{I}\left(K_{a c}^{I} K_{b d}^{I}-K_{b c}^{I} K_{a d}^{I}\right), \quad I=1, \ldots, N .
$$

Contracting this relation and taking into account Eq. (3), one obtains the equation

$\epsilon_{I}\left(R-2 \Lambda_{I}\right)+K_{a b}^{I} K_{I}^{a b}-K_{I}^{2} \equiv \epsilon_{I}\left(R-2 \Lambda_{I}\right)+S_{a b}^{I} S_{I}^{a b}-\frac{1}{3} S_{I}^{2}=0, \quad I=1, \ldots, N,(8)$ which is valid on all sides of the brane, and which we expressed in terms of $S_{a b}^{I}=$ $K_{a b}^{I}-h_{a b} K^{I}$ and $S^{I}=h^{a b} S_{a b}^{I}$. This is the well-known constraint equation on the brane from the viewpoint of the gravitational dynamics in the five-dimensional bulk.

Our aim is to derive the resulting cosmological equation on the brane. One could solve this problem by considering embedding of the brane in the bulk spaces under consideration and calculating the corresponding extrinsic curvatures. However, in a cosmological setup, one can integrate the constraint equations (8) directly on the brane. First, we note that, for any tensor on the brane $T_{a b}$ which is covariantly conserved, i.e., $D_{a} T^{a}{ }_{b}=0$, in the cosmological setup

$$
T^{0}{ }_{0}=\beta(t), \quad T^{\mu}{ }_{\nu}=\delta^{\mu}{ }_{\nu} q(t), \quad \mu, \nu=1,2,3,
$$

one can easily verify the following relation:

$$
T_{a b} T^{a b}-\frac{1}{3} T^{2}=\frac{1}{3 a^{4} H} \frac{d}{d t}\left(a^{4} \beta^{2}\right) .
$$

In view of the conservation equation, the function $q(t)$ in (9) is uniquely expressed through $\beta(t)$. Then, setting

$$
S_{I 0}^{0}=-3 \beta_{I}(t), \quad I=1, \ldots, N,
$$

and using property (10), valid for all these tensors in view of the conservation equation (5), we can integrate Eq. (8) with the result

$$
\epsilon_{I}\left(H^{2}+\frac{\kappa}{a^{2}}-\frac{\Lambda_{I}}{6}\right)=\beta_{I}^{2}+\frac{\epsilon_{I} C_{I}}{a^{4}}, \quad I=1, \ldots, N,
$$

\footnotetext{
${ }^{\mathrm{b}}$ Those interested in the derivation of (4) may look into the appendices of Refs. 26, 28
} 
where $C_{I}$ are integration constants. The zero-zero component of Eq. (4) gives

$$
H^{2}+\frac{\kappa}{a^{2}}=\frac{\rho+\sigma}{3 m^{2}}+\frac{1}{m^{2}} \sum_{I=1}^{N} \epsilon_{I} M_{I}^{3} \beta_{I} .
$$

Substituting $\beta_{I}$ found from Eq. (12) into this equation, we obtain our main result:

$$
H^{2}+\frac{\kappa}{a^{2}}=\frac{\rho+\sigma}{3 m^{2}}+\frac{1}{m^{2}} \sum_{I=1}^{N} \zeta_{I} M_{I}^{3}\left[\epsilon_{I}\left(H^{2}+\frac{\kappa}{a^{2}}-\frac{\Lambda_{I}}{6}-\frac{C_{I}}{a^{4}}\right)\right]^{1 / 2},
$$

where $\zeta_{I}= \pm 1$ corresponds to the possibility of different signs in the solution for $\beta_{I}$ from Eq. (12). Physically, these signs correspond to the two possible ways of bounding each of the spaces $\mathcal{M}_{I}$ by the brane.

The integration constants $C_{I}$ generalize the so-called "dark radiation" contribution to the dynamics of the brane. In the case where the brane is a boundary of $N$ independent bulk spaces, there are exactly $N$ such independent integration constants. If nonzero, they reflect the existence of black holes in the corresponding bulk spaces.

\section{The case of two bulk spaces}

In this section, we consider in detail the case where the brane is just embedded in one bulk space, so that $N=2$, i.e., there are only two "sides" of the brane. Equation (4) involves the tensors $S_{a b}^{I}$ which are constructed from the tensors of extrinsic curvature of the brane, so this equation is not closed with respect to the intrinsic evolution on the brane. However, using (8), it is possible to obtain a system of scalar equations which involves only four-dimensional fields on the brane. Following, with

slight modifications, the procedure first adopted in Ref. 21 for the particular case $M_{1}=M_{2}$ and $\epsilon_{1}=\epsilon_{2}=1$, we introduce the tensors

$$
\Sigma_{a b}=\epsilon_{1} M_{1}^{3} S_{a b}^{(1)}+\epsilon_{2} M_{2}^{3} S_{a b}^{(2)}, \quad \Delta_{a b}=\epsilon_{1} M_{1}^{3} S_{a b}^{(1)}-\epsilon_{2} M_{2}^{3} S_{a b}^{(2)} .
$$

Rewriting (8) in terms of $\Sigma_{a b}$ and $\Delta_{a b}$, we easily obtain the following closed system of gravitational equations on the brane:

$$
\begin{gathered}
\left(\Sigma_{a b} \Sigma^{a b}-\frac{1}{3} \Sigma^{2}\right)+\left(\Delta_{a b} \Delta^{a b}-\frac{1}{3} \Delta^{2}\right) \\
+2\left(\epsilon_{1} M_{1}^{6}+\epsilon_{2} M_{2}^{6}\right) R-4\left(\epsilon_{1} M_{1}^{6} \Lambda_{1}+\epsilon_{2} M_{2}^{6} \Lambda_{2}\right)=0, \\
\left(\Sigma_{a b} \Delta^{a b}-\frac{1}{3} \Sigma \Delta\right)+\left(\epsilon_{1} M_{1}^{6}-\epsilon_{2} M_{2}^{6}\right) R-2\left(\epsilon_{1} M_{1}^{6} \Lambda_{1}-\epsilon_{2} M_{2}^{6} \Lambda_{2}\right)=0, \\
D_{a} \Delta^{a}{ }_{b}=0,
\end{gathered}
$$

where $\Sigma=\Sigma_{a b} h^{a b}, \Delta=\Delta_{a b} h^{a b}$, and $\Sigma_{a b}$ is given by

$$
\Sigma_{a b}=m^{2} G_{a b}+\sigma h_{a b}-\tau_{a b}
$$

in view of (4). 
This system of equations is to be solved for the metric and matter fields and for the symmetric tensor field $\Delta_{a b}$ on the brane. It constitutes the main system of closed scalar equations on the brane, which arises in the absence of any information and/or boundary conditions for the brane-bulk system. For the particular case $M_{1}=M_{2}$ and $\epsilon_{1}=\epsilon_{2}=1$, this system of equations was first obtained in Ref. 21,

In what follows, we consider the cosmological implications of system (16)-(19) for the homogeneous and isotropic cosmological model with the cosmological time $t$, scale factor $a(t)$, energy density $\rho(t)$, and pressure $p(t)$. Under these conditions, we set the tensor $\Delta_{a b}$ to be homogeneous and isotropic as well:

$$
\Delta^{0}{ }_{0}=-\beta(t), \quad \Delta^{\mu}{ }_{\nu}=\delta^{\mu}{ }_{\nu} q(t), \quad \mu, \nu=1,2,3 .
$$

Using (6) and (18), it is easy to calculate the quantities

$$
\begin{gathered}
\Sigma_{a b} \Sigma^{a b}-\frac{1}{3} \Sigma^{2}=-\frac{1}{3 a^{4} H} \frac{d}{d t}\left[a^{2}\left(3 m^{2} \chi-\rho-\sigma\right)\right]^{2}, \\
\Delta_{a b} \Delta^{a b}-\frac{1}{3} \Delta^{2}=-\frac{1}{3 a^{4} H} \frac{d}{d t}\left(a^{4} \beta^{2}\right), \\
\Sigma_{a b} \Delta^{a b}-\frac{1}{3} \Sigma \Delta=-\frac{1}{3 a^{4} H} \frac{d}{d t}\left[a^{4} \beta\left(3 m^{2} \chi-\rho-\sigma\right)\right],
\end{gathered}
$$

where $H \equiv \dot{a} / a$ is the Hubble parameter,

$$
\chi \equiv H^{2}+\frac{\kappa}{a^{2}},
$$

and $\kappa=0, \pm 1$ corresponds to the sign of the spatial curvature of the model.

Since the expressions $a^{3} \dot{a}$ and $a^{3} \dot{a} R$ are also total derivatives, equations (16) and (17) can be integrated with the result

$$
\begin{gathered}
m^{4}\left(\chi-\frac{\rho+\sigma}{3 m^{2}}\right)^{2}+\frac{\beta^{2}}{9}=2\left(\epsilon_{1} M_{1}^{6}+\epsilon_{2} M_{2}^{6}\right) \chi-\frac{1}{3}\left(\epsilon_{1} M_{1}^{6} \Lambda_{1}+\epsilon_{2} M_{2}^{6} \Lambda_{2}\right)-\frac{C}{a^{4}}, \\
\beta\left(3 m^{2} \chi-\rho-\sigma\right)=9\left(\epsilon_{1} M_{1}^{6}-\epsilon_{2} M_{2}^{6}\right) \chi-\frac{3}{2}\left(\epsilon_{1} M_{1}^{6} \Lambda_{1}-\epsilon_{2} M_{2}^{6} \Lambda_{2}\right)-\frac{9 E}{a^{4}},
\end{gathered}
$$

where $C$ and $E$ are integration constants. Then, eliminating $\beta$ from (26) and using (25), we finally obtain

$$
\begin{aligned}
& m^{4}\left(H^{2}+\frac{\kappa}{a^{2}}-\frac{\rho+\sigma}{3 m^{2}}\right)^{2}-2\left(\epsilon_{1} M_{1}^{6}+\epsilon_{2} M_{2}^{6}\right)\left(H^{2}+\frac{\kappa}{a^{2}}\right) \\
& +\frac{1}{3}\left(\epsilon_{1} M_{1}^{6} \Lambda_{1}+\epsilon_{2} M_{2}^{6} \Lambda_{2}\right)+\frac{C}{a^{4}} \\
& +\left[\frac{\left(\epsilon_{1} M_{1}^{6}-\epsilon_{2} M_{2}^{6}\right)\left(H^{2}+\kappa / a^{2}\right)-\left(\epsilon_{1} M_{1}^{6} \Lambda_{1}-\epsilon_{2} M_{2}^{6} \Lambda_{2}\right) / 6-E / a^{4}}{m^{2}\left(H^{2}+\kappa / a^{2}\right)-(\rho+\sigma) / 3}\right]^{2}=0 .
\end{aligned}
$$

This is our main result as regards cosmology without $Z_{2}$ symmetry in the bulk. It could also be obtained directly from (14) with $I=2$. This equation in its general form is rather complicated, so it is useful to consider some partial cases. The general case $\epsilon_{1}=\epsilon_{2}=+1$ will be investigated in more detail elsewhere. 
3.1. $\epsilon_{1}=\epsilon_{2}=+1$ and $\Lambda_{1}=\Lambda_{2}$ or $M_{1}=M_{2}$

We begin with the case $\Lambda_{1}=\Lambda_{2}=\Lambda$ and also set $\epsilon_{1}=\epsilon_{2}=+1$. Then Eq. (27) becomesc

$$
\begin{aligned}
m^{4}\left(H^{2}-\right. & \left.\frac{\rho+\sigma}{3 m^{2}}\right)^{2}-2\left(M_{1}^{6}+M_{2}^{6}\right)\left(H^{2}-\frac{\Lambda}{6}\right)+\frac{C}{a^{4}} \\
& +\left[\frac{\left(M_{1}^{6}-M_{2}^{6}\right)\left(H^{2}-\Lambda / 6\right)-E / a^{4}}{m^{2} H^{2}-(\rho+\sigma) / 3}\right]^{2}=0 .
\end{aligned}
$$

The last term on the right-hand side of this equation represents the difference between our model and standard $Z_{2}$-symmetric cosmology. Setting additionally

$M_{1}=M_{2}=M$ and $E=0$, we obtain the well-known result of the $Z_{2}$-symmetric case $17 \mid 31$

$$
m^{4}\left(H^{2}-\frac{\rho+\sigma}{3 m^{2}}\right)^{2}=4 M^{6}\left(H^{2}-\frac{\Lambda}{6}-\frac{C_{1}}{a^{4}}\right), \quad C_{1}=\frac{C}{4 M^{6}} .
$$

This equation can easily be solved with respect to the Hubble parameter, giving two branches $\underline{415}$

$$
H^{2}=\frac{\rho+\sigma}{3 m^{2}}+\frac{2 M^{6}}{m^{4}} \pm \frac{2 M^{6}}{m^{4}}\left[1+\frac{m^{4}}{M^{6}}\left(\frac{\rho+\sigma}{3 m^{2}}-\frac{\Lambda}{6}-\frac{C_{1}}{a^{4}}\right)\right]^{1 / 2} .
$$

Setting $\epsilon_{1}=\epsilon_{2}=\epsilon$ and $M_{1}=M_{2}=M$ but different $\Lambda_{1}$ and $\Lambda_{2}$ in Eq. (27), we obtain

$$
\begin{aligned}
m^{4}\left(H^{2}-\frac{\rho+\sigma}{3 m^{2}}\right)^{2}= & 4 \epsilon M^{6}\left(H^{2}-\frac{\Lambda_{1}+\Lambda_{2}}{12}-\frac{\epsilon C_{1}}{a^{4}}\right) \\
& -\frac{M^{12}}{36}\left[\frac{\Lambda_{1}-\Lambda_{2}+E_{1} / a^{4}}{m^{2} H^{2}-(\rho+\sigma) / 3}\right]^{2},
\end{aligned}
$$

where $C_{1}$ is defined in (29), and

$$
E_{1}=\frac{6 E}{M^{6}}
$$

Equation (31) coincides with the result of Ref. 21,

\section{2. $\Lambda_{1}=\Lambda_{2}$ and $M_{1}=M_{2}$}

Another interesting possibility is to take all the constants of the theory equal on the two sides of the brane (i. e., $\Lambda_{1}=\Lambda_{2}=\Lambda$ and $M_{1}=M_{2}=M$ ), but let $E \neq 0$. This means that the $Z_{2}$ symmetry is broken only by the difference of the masses of black holes on the two sides of the brane. After some redefinitions, in this case, from (27), we get (again, for simplicity, taking $\epsilon_{1}=\epsilon_{2}=+1$ )

$$
m^{4} X^{4}-4 M^{6} X^{3}-4 M^{6}\left(\frac{\rho+\sigma}{3 m^{2}}-\frac{\Lambda}{6}-\frac{C_{1}}{a^{4}}\right) X^{2}+\frac{E^{2}}{m^{4} a^{8}}=0,
$$

${ }^{\mathrm{c}}$ Here and below, for notational simplicity, we omit the spatial curvature term $\kappa / a^{2}$. It can easily be recovered by the substitution $H^{2} \rightarrow H^{2}+\kappa / a^{2}$ in all formulas. 
where $X$ stands for the expression $H^{2}-(\rho+\sigma) / 3 m^{2}$. This result is very similar to that obtained in Ref. 20, where this equation was solved in the limit of small but nonzero $m$. In the limit $m \rightarrow 0$, equation (33) reduces to the equation

$$
H^{2}=\frac{\Lambda}{6}+\frac{C_{1}}{a^{4}}+\frac{(\rho+\sigma)^{2}}{36 M^{6}}+\frac{9 E^{2}}{4 a^{8}(\rho+\sigma)^{2}},
$$

which was under consideration in Refs. 17, 18, 19.

\section{3. $m=0$ and $\epsilon_{1}=\epsilon_{2}=+1$}

The limit $m=0$ was thoroughly investigated in the previous literature. Taking this limit in Eq. (27) and keeping $\epsilon_{1}=\epsilon_{2}=+1$, one obtains

$$
\begin{aligned}
H^{2} & =\frac{M_{1}^{6} \Lambda_{1}-M_{2}^{6} \Lambda_{2}}{6\left(M_{1}^{6}-M_{2}^{6}\right)}+\frac{E_{2}}{a^{4}}+\frac{M_{1}^{6}+M_{2}^{6}}{9\left(M_{1}^{6}-M_{2}^{6}\right)^{2}}(\rho+\sigma)^{2} \\
& \pm \frac{2 M_{1}^{3} M_{2}^{3}}{9\left(M_{1}^{6}-M_{2}^{6}\right)^{2}}(\rho+\sigma)\left[(\rho+\sigma)^{2}+\frac{3}{2}\left(\Lambda_{1}-\Lambda_{2}\right)\left(M_{1}^{6}-M_{2}^{6}\right)-\frac{C_{2}}{a^{4}}\right]^{1 / 2},
\end{aligned}
$$

where

$$
C_{2}=\frac{9}{4}\left[\left(\frac{1}{M_{2}^{6}}-\frac{1}{M_{1}^{6}}\right) C-3 E\left(\frac{1}{M_{2}^{6}}+\frac{1}{M_{1}^{6}}\right)\right], \quad E_{2}=\frac{3 E}{2\left(M_{1}^{6}-M_{2}^{6}\right)} .
$$

This equation was studied by Padilla ${ }^{32}$ He noticed that, in a certain range of parameters, this model cosmologically behaves as the $Z_{2}$-symmetric braneworld

model with induced gravity. Specifically, with the upper sign in (35) and with the assumptions

$$
\frac{\left|C_{2}\right|}{a^{4}} \ll\left(\Lambda_{1}-\Lambda_{2}\right)\left(M_{1}^{6}-M_{2}^{6}\right),
$$

in which the right-hand side is assumed to be positive, and

$$
(\rho+\sigma) \ll \frac{M_{1}^{3} M_{2}^{3}}{M_{1}^{6}+M_{2}^{6}} \sqrt{\left(\Lambda_{1}-\Lambda_{2}\right)\left(M_{1}^{6}-M_{2}^{6}\right)},
$$

equation (35) reduces to

$$
H^{2}=\frac{\rho+\sigma}{3 m_{\mathrm{eff}}^{2}}+\frac{\Lambda_{\mathrm{eff}}}{6}+\frac{E_{2}}{a^{4}}
$$

where

$$
\begin{gathered}
\Lambda_{\text {eff }}=\frac{M_{1}^{6} \Lambda_{1}-M_{2}^{6} \Lambda_{2}}{M_{1}^{6}-M_{2}^{6}}, \\
m_{\text {eff }}^{2}=\sqrt{\frac{3\left(M_{1}^{6}-M_{2}^{6}\right)^{3}}{2 M_{1}^{6} M_{2}^{6}\left(\Lambda_{1}-\Lambda_{2}\right)}},
\end{gathered}
$$

which has the form of the Friedmann equation with cosmological constant and "dark radiation" [the last term in (39)] ]. 


\section{4. $M_{1}=M_{2}$ and $\epsilon_{1}=-\epsilon_{2}=+1$}

The structure of Eq. (27) allows one to consider the case $M_{1}=M_{2}=M$ with the (somewhat exotic) condition $\epsilon_{1}=-\epsilon_{2}=+1$. In this case, we have

$$
\begin{aligned}
m^{4}\left(H^{2}-\frac{\rho+\sigma}{3 m^{2}}\right)^{2} & +\frac{4 M^{12}}{m^{4}}\left[\frac{H^{2}-\left(\Lambda_{1}+\Lambda_{2}\right) / 12-E_{1} / 12 a^{4}}{H^{2}-(\rho+\sigma) / 3 m^{2}}\right]^{2} \\
& =4 M^{6}\left(\frac{\Lambda_{2}-\Lambda_{1}}{12}-\frac{C_{1}}{a^{4}}\right)
\end{aligned}
$$

where $E_{1}$ is defined in (32), and $C_{1}$ in (29). In the limit $m \rightarrow 0$, this equation reduces to

$$
H^{2}=\frac{\Lambda_{1}+\Lambda_{2}}{12}+\frac{E_{1}}{12 a^{4}} \pm \frac{(\rho+\sigma)}{3 M^{3}}\left[\frac{\Lambda_{2}-\Lambda_{1}}{12}-\frac{C_{1}}{a^{4}}-\frac{(\rho+\sigma)^{2}}{36 M^{6}}\right]^{1 / 2} .
$$

With the upper sign and for $\Lambda_{2} \gg \Lambda_{1}$, this again has the form of (39) with different effective constants $m_{\text {eff }}$ and $\Lambda_{\text {eff }}$.

\section{Conclusion}

In this letter, we derived a system of cosmological equations for a braneworld which is a multivolume junction, i.e., a boundary of $N$ bulk spaces with natural junction conditions, without assuming any symmetry between the bulk spaces. In particular, the values of the fundamental constants, and even the signatures of the extra dimension, could be different on different sides of the brane. After that, we studied in more detail the usual case of two bulk spaces, and obtained the closed system of scalar gravitational equations (16)-(18) describing the braneworld in the absence of the $Z_{2}$ symmetry of reflection of the bulk with respect to the brane, with different fundamental constants in the bulk, and even with possibly different time signature of the fifth dimension on the two sides of the brane. We then derived equation (27) describing the cosmological evolution of such a brane and considered several special cases of this general situation.

\section{Acknowledgments}

Yu. S. and A. V. were supported in part by the "Cosmomicrophysics" programme and by the Program of Fundamental Research of the Physics and Astronomy Division of the National Academy of Sciences of Ukraine, by grant No. F16-457-2007 of the State Foundation of Fundamental Research of Ukraine, and by the INTAS grant No. 05-1000008-7865. L. N. G. acknowledges financial support from COLCIENCIAS (Colombia).

\section{References}

1. R. Maartens, Living Rev. Rel. 7, 7 (2004) arXiv:gr-qc/0312059. 
2. V. Sahni, "Cosmological Surprises from Braneworld models of Dark Energy," in: Proceedings of the 14th Workshop on General Relativity and Gravitation (JGRG14), Kyoto University, Japan, 29 November - 3 December, 2004, Ed. by W. Hikida, M. Sasaki, T. Tanaka, and T. Nakamura, pp. 95-115 arXiv:astro-ph/0502032.

3. K. Koyama, "The cosmological constant and dark energy in braneworlds," arXiv:0706.1557.

4. V. Sahni and Yu. Shtanov, JCAP 0311, 014 (2003) arXiv:astro-ph/0202346.

5. V. Sahni and Yu. V. Shtanov, IJMP D 11, 1 (2002) arXiv:gr-qc/0205111].

6. V. Sahni and Yu. Shtanov, Phys. Rev. D 71, 084018 (2005) arXiv:astro-ph/0410221.

7. V. Sahni, Yu. Shtanov, and A. Viznyuk, JCAP 0512, 005 (2005) arXiv:astro-ph/0505004.

8. A. Viznyuk and Yu. Shtanov, Phys. Rev. D 76, 064009 (2007) arXiv:0706.0649.

9. P. Kraus, JHEP 9912, 011 (1999) arXiv:hep-th/9910149.

10. H. Stoica, H. Tye, and I. Wasserman, Phys. Lett. B 482, 205 (2000) arXiv:hep-th/0004126.

11. P. Bowcock, C. Charmousis, and R. Gregory, Class. Quantum Grav. 17, 4745 (2000) arXiv:hep-th/0007177.

12. B. Carter and J.-P. Uzan, Nucl. Phys. B 406, 45 (2001) arXiv:gr-qc/0101010

13. R. A. Battye, B. Carter, A. Mennim, and J.-P. Uzan, Phys. Rev. D 64, 124007 (2001) arXiv:hep-th/0105091

14. N. Deruelle and T. Doležel, Phys. Rev. D 62, 103502 (2000) arXiv:gr-qc/0004021.

15. W. B. Perkins, Phys. Lett. B 504, 28 (2001) arXiv:gr-qc/0010053.

16. G. Kofinas, JHEP 0108, 034 (2001) arXiv:hep-th/0108013.

17. H. Collins and R. Holdom, Phys. Rev. D 62, 105009 (2000) arXiv:hep-ph/0003173.

18. D. Ida, JHEP 0009, 014 (2000) arXiv:gr-qc/9912002.

19. A. C. Davis, I. Vernon, S. C. Davis, and W. B. Perkins, Phys. Lett. B 504, 254 (2001) arXiv:hep-ph/0008132.

20. N. J. Kim, H. W. Lee, and Y. S. Myung, Phys. Lett. B 504, 323 (2001) arXiv:hep-th/0101091.

21. Yu. V. Shtanov, Phys. Lett. B 541, 177 (2002) arXiv:hep-ph/0108153.

22. K. Koyama and K. Koyama, Phys. Rev. D 72, 043511 (2005) arXiv:hep-th/0501232.

23. M. Chaichian and A. B. Kobakhidze, Phys. Lett. B 488, 117 (2000) arXiv:hep-th/0003269.

24. Z. Berezhiani, M. Chaichian, A. B. Kobakhidze, and Z.-H. Yu, Phys. Lett. B 517, 387 (2001) arXiv:hep-th/0102207.

25. Yu. Shtanov and V. Sahni, Phys. Lett. B 557, 1 (2003) arXiv:gr-qc/0208047.

26. P. I. Fomin and Yu. V. Shtanov, Class. Quantum Grav. 19, 3139 (2002) arXiv:hep-th/0008183.

27. R. M. Wald, General Relativity (The University of Chicago Press, Chicago, 1984).

28. Yu. V. Shtanov, "On brane-world cosmology," arXiv:hep-th/0005193.

29. P. Binetruy, C. Deffayet, and D. Langlois, Nucl. Phys. B 565, 26 (2000) arXiv:hep-th/9905012.

30. T. Shiromizu, K. Maeda, and M. Sasaki, Phys. Rev. D 62, 024012 (2000) arXiv:gr-qc/9910076.

31. C. Deffayet, Phys. Lett. B 502, 199 (2001) arXiv:hep-th/0010186].

32. A. Padilla, Class. Quantum Grav. 22, 681 (2005) [arXiv:hep-th/0406157]. 